\title{
Ellerman bombs and UV bursts: transient events in chromospheric current sheets ${ }^{\star}$
}

\author{
V. Hansteen ${ }^{1,2}$, A. Ortiz ${ }^{1,2}$, V. Archontis ${ }^{3}$, M. Carlsson ${ }^{1,2}$, T. M. D. Pereira ${ }^{1,2}$, and J. P. Bjørgen ${ }^{4,1}$ \\ ${ }^{1}$ Rosseland Centre for Solar Physics, University of Oslo, PO Box 1029, Blindern 0315, Oslo, Norway \\ e-mail: viggoh@astro.uio.no \\ 2 Institute of Theoretical Astrophysics, University of Oslo, PO Box 1029, Blindern 0315, Oslo, Norway \\ 3 School of Mathematics and Statistics, St. Andrews University, St. Andrews KY169SS, UK \\ ${ }^{4}$ Institute for Solar Physics, Department of Astronomy, Stockholm University, Albanova University Centre, 10691 Stockholm, \\ Sweden
}

Received 27 February 2019 / Accepted 7 April 2019

\begin{abstract}
Context. Ellerman bombs (EBs), observed in the photospheric wings of the $\mathrm{H} \alpha$ line, and UV bursts, observed in the transition region $\mathrm{Si}$ Iv line, are both brightenings related to flux emergence regions and specifically to magnetic flux of opposite polarity that meet in the photosphere. These two reconnection-related phenomena, nominally formed far apart, occasionally occur in the same location and at the same time, thus challenging our understanding of reconnection and heating of the lower solar atmosphere.

Aims. We consider the formation of an active region, including long fibrils and hot and dense coronal plasma. The emergence of a untwisted magnetic flux sheet, injected $2.5 \mathrm{Mm}$ below the photosphere, is studied as it pierces the photosphere and interacts with the preexisting ambient field. Specifically, we aim to study whether EBs and UV bursts are generated as a result of such flux emergence and examine their physical relationship.

Methods. The Bifrost radiative magnetohydrodynamics code was used to model flux emerging into a model atmosphere that contained a fairly strong ambient field, constraining the emerging field to a limited volume wherein multiple reconnection events occur as the field breaks through the photosphere and expands into the outer atmosphere. Synthetic spectra of the different reconnection events were computed using the 1.5D RH code and the fully 3D MULTI3D code.

Results. The formation of UV bursts and EBs at intensities and with line profiles that are highly reminiscent of observed spectra are understood to be a result of the reconnection of emerging flux with itself in a long-lasting current sheet that extends over several scale heights through the chromosphere. Synthetic spectra in the $\mathrm{H} \alpha$ and Si Iv $139.376 \mathrm{~nm}$ lines both show characteristics that are typical of the observations. These synthetic diagnostics suggest that there are no compelling reasons to assume that UV bursts occur in the photosphere. Instead, EBs and UV bursts are occasionally formed at opposite ends of a long current sheet that resides in an extended bubble of cool gas.
\end{abstract}

Key words. magnetohydrodynamics - magnetic reconnection - Sun: atmosphere - Sun: chromosphere - Sun: magnetic fields Sun: UV radiation

\section{Introduction}

Emerging flux regions are host to a large variety of transient phenomena that are driven by the interaction of the emerging field with the photospheric, chromospheric, and coronal plasma, the preexisting ambient field, and of the field with itself. Such interactions are a necessary part of the rise of the field into the corona and the forming magnetic field of the active region; the rising field carries with it considerable mass, which must fall back to and through the photosphere in order to allow the field to attain coronal heights. Reconnection plays an important role in this process, cutting field lines so that dense material can fall while at the same time alleviating the weight on the field lines and allowing them to form the longer loops that make up the active region corona and chromosphere of the active region (see, e.g., Pariat et al. 2004).

These dynamical phenomena include Ellerman bombs (EB hereafter, Ellerman 1917) and UV bursts (Peter et al. 2014), which both occur in the vicinity of merging or cancelling photospheric fields of opposite polarities. Ellerman bombs are first

\footnotetext{
* The movie associated to Fig. 3 is available at https://www. aanda.org
}

and foremost recognized by strong emission in the wings of $\mathrm{H} \alpha$, with little or no visible signal in the line core. This indicates that EBs are formed at photospheric or upper photospheric heights, at most some few hundred kilometers above the photosphere. As observed in spectroheliograms in the wings of $\mathrm{H} \alpha$, they appear as flame-like structures that jut out of the photosphere when seen toward the limb (Watanabe et al. 2011). The primary signature of UV bursts is found in small $\left(\sim 1^{\prime \prime}\right)$ extreme brightenings in the Si Iv lines that have been observed with the Interface Region Imaging Spectrograph (IRIS; De Pontieu et al. 2014) to be as much as two to three orders of magnitude brighter than the average emission. This brightening is accompanied by large line broadening, of order $200 \mathrm{~km} \mathrm{~s}^{-1}$ or more, and importantly, superimposed absorption features by lines associated with much cooler temperatures such as from $\mathrm{Ni}$ II and $\mathrm{Fe}_{\text {II }}$ lines. UV bursts and EBs are also found at sunspot light bridges (e.g., Toriumi et al. 2015) and in moving magnetic features (e.g., Gupta \& Tripathi 2015).

The observed relationship between EBs and UV bursts has recently been the focus of debate and several papers, see Vissers et al. (2015), Kim et al. (2015), Grubecka et al. (2016), Tian et al. (2016), and Young et al. (2018), and references cited 
therein, and most recently, Ortiz et al. (in prep.). The question is whether EBs and UV bursts are connected, and if so, how they are related. The height at which the plasma temperature becomes high enough to emit radiation in the Si Iv lines also needs to be determined. Peter et al. (2014) have speculated on the possibility of a relationship between UV bursts and EBs. These authors were unable to conclude whether UV bursts and EBs were the same phenomena or not. Judge (2015) suggested that the Si IV emission was more naturally explained as stemming from the chromosphere and a result of Alfvénic turbulence. Vissers et al. (2015) noted that all $\mathrm{H} \alpha$ fitting exercises show that EBs represent temperature enhancements of the low standard model chromosphere by at most a few thousand Kelvin ot less. In addition, they claim that Si Iv intensities with the observed properties cannot be obtained from any of these models. On the other hand, based on O IV emission, Mn I absorption in the wings of $\mathrm{Mg}$ II, enhanced emission in the $\mathrm{Mg}$ II wings but not core, deep absorption in Ni II lines, and compact brightenings in the AIA $170 \mathrm{~nm}$ passband, Tian et al. (2016) concluded that the UV bursts that occur in connection with EBs are formed in the photosphere. While further noting that other UV bursts may be formed in the chromosphere. Fang et al. (2017) point out that the observed $\mathrm{H} \alpha$ emission cannot be reproduced using non-local thermal equilibrium (non-LTE) semi-empirical modeling if the temperature is above $10000 \mathrm{~K}$. They suggested that the coincidence of EBs and UV bursts could be due a projection effect. Furthermore, Ortiz et al. (in prep.), for example, found that EBs and UV bursts can be cospatial and cotemporal, but can also occur independently of each other in both time and location. The occurrence of cospatial and cotemporal EBs and UV bursts in the same spectral image is between $10 \%$ and $20 \%$ of all observed $\mathrm{H} \alpha$ brightenings in that image. When these two reconnection events do occur together, they may do so nearly simultaneously or with a delay of a few minutes; the UV burst shows a more rapid rise and fall in intensity and is more short lived than the cospatial EB.

The question then is whether and how models can naturally reproduce the required conditions to generate EBs and UV bursts in the same location and at the same time. Modeling reproduces the diagnostic signatures of EBs and UV bursts as a result of large angle reconnection, essentially of fields oriented in diametrically opposite directions. EBs result when the reconnection occurs in the upper photosphere with temperatures some 1000$3000 \mathrm{~K}$ higher than ambient (e.g., Rutten et al. 2013), while UV bursts are reproduced when the reconnection is located higher in the chromosphere and entails much higher temperatures than those found in EB models, at least high enough to ionize silicon three times: $20 \mathrm{kK}$ if in dense photospheric material, $80 \mathrm{kK}$ at lower densities, as pointed out by Rutten (2016). It may be possible to generate temperatures as high as $25 \mathrm{kK}$ through reconnection in the upper photosphere or lower chromosphere, as shown by $\mathrm{Ni}$ et al. $(2016,2018)$.

Priest et al. (2018) and Syntelis et al. (2019) pointed out in a theoretical work that flux cancellation can cause simultaneous reconnection at different heights in the solar atmosphere. Hansteen et al. (2017) found that both EBs and UV bursts were generated in a model where a flux sheet was injected some $2.5 \mathrm{Mm}$ below the photosphere and allowed to emerge through the photosphere into an essentially magnetic field free corona. Upon reaching the photosphere, no longer buoyant, the field stalled, but eventually emerged in locations of strong field, filling the chromosphere with expanding cool bubbles. These bubbles pushed the original weak-field outer atmosphere aside, filling a large area in height with cool gas. As the bubbles grew horizontally, they came into contact with each other, typically with oppositely oriented fields, and formed current sheets. These locations were the sites of EBs and UV bursts.

However, in that model no colocated or cotemporal EBs and UV bursts were produced. Rather, it was found that the high velocities and temperatures needed to produce UV bursts occurred several chromospheric scale heights above the photosphere, while EBs were confined to locations just above the photosphere. In this paper a model very similar to that described in Hansteen et al. (2017) is considered, but instead of a weak 0.1 Gauss slanted field, a stronger preexisting outer atmospheric field (average field strength 2 Gauss at $10 \mathrm{Mm}$ ) that is oriented at an angle to the injected emerging field is employed. Thus, in addition to improving the numerical resolution, the impetus of this work is the expectation that the preexisting ambient field helps contain and confine the emerging field. This should give rise to large angle reconnection and thus powerful coronal heating as the fields eventually interact, forming a typical realistic active region chromosphere covered by a hot corona. We do indeed find the formation of long chromospheric fibrils in this model, as well as significant coronal heating. The average temperature at $10 \mathrm{Mm}$ initially is $2 \mathrm{MK}$, but rises in the span of $30 \mathrm{~min}$ to almost $10 \mathrm{MK}$ before falling again over the next hour to the $4-5 \mathrm{MK}$ that are typical of active regions (Warren et al. 2012). In addition, we also find that both EBs and UV bursts are produced. Occasionally and critically, we find that these phenomena are found to be both colocated and cotemporal, as observed, for instance, by Vissers et al. (2015), Tian et al. (2016), and Ortiz et al. (in prep.). Our simulations seem to indicate that the concurrence of EBs and UV bursts are due to a long vertical or nearly vertical current sheet with intensive reconnection that stretches from the upper photosphere, where EB signatures are generated, to several thousand kilometers upward. UV bursts are the result of reconnection across several chromospheric scale heights well above the photosphere. Line and continuum absorption is provided by cool gas that is carried into a vastly expanded chromosphere as the emerging field breaks through the photosphere and rises to fill the nascent active region corona.

\section{Methods}

\subsection{Initial model}

The initial model we used for this study is a version of the publicly available Bifrost model (Carlsson et al. 2016) with higher resolution that covers the same spatial extent and has roughly the same magnetic topology. This new model was run for roughly one hour solar time and is designed to have an effective temperature of $T_{\text {eff }} \approx 5780 \mathrm{~K}$, convective energy transport below the photosphere, and radiative losses maintained by the injection of entropy at the bottom boundary. The outer atmosphere is maintained by acoustic shocks generated from motions in the convection zone and by the interaction of convective motions and the magnetic field.

The average field strength in the photosphere is 50 Gauss originally, and at the onset of the run, the vertical component of the photospheric field is concentrated in two amorphous regions, positive polarities between $x=[6,10] \mathrm{Mm}$ and $y=$ $[5,15] \mathrm{Mm}$, and negative polarities some $10 \mathrm{Mm}$ distant between $x=[15,19] \mathrm{Mm}$ and $y=[12,17] \mathrm{Mm}$, as shown in the left panel of Fig. 1. Both polarities have maximum strengths of about 1500 Gauss in the photosphere, and chromospheric and coronal field lines stretch between them. Hints of these field lines are visible in the temperature structure of the lower corona shown 

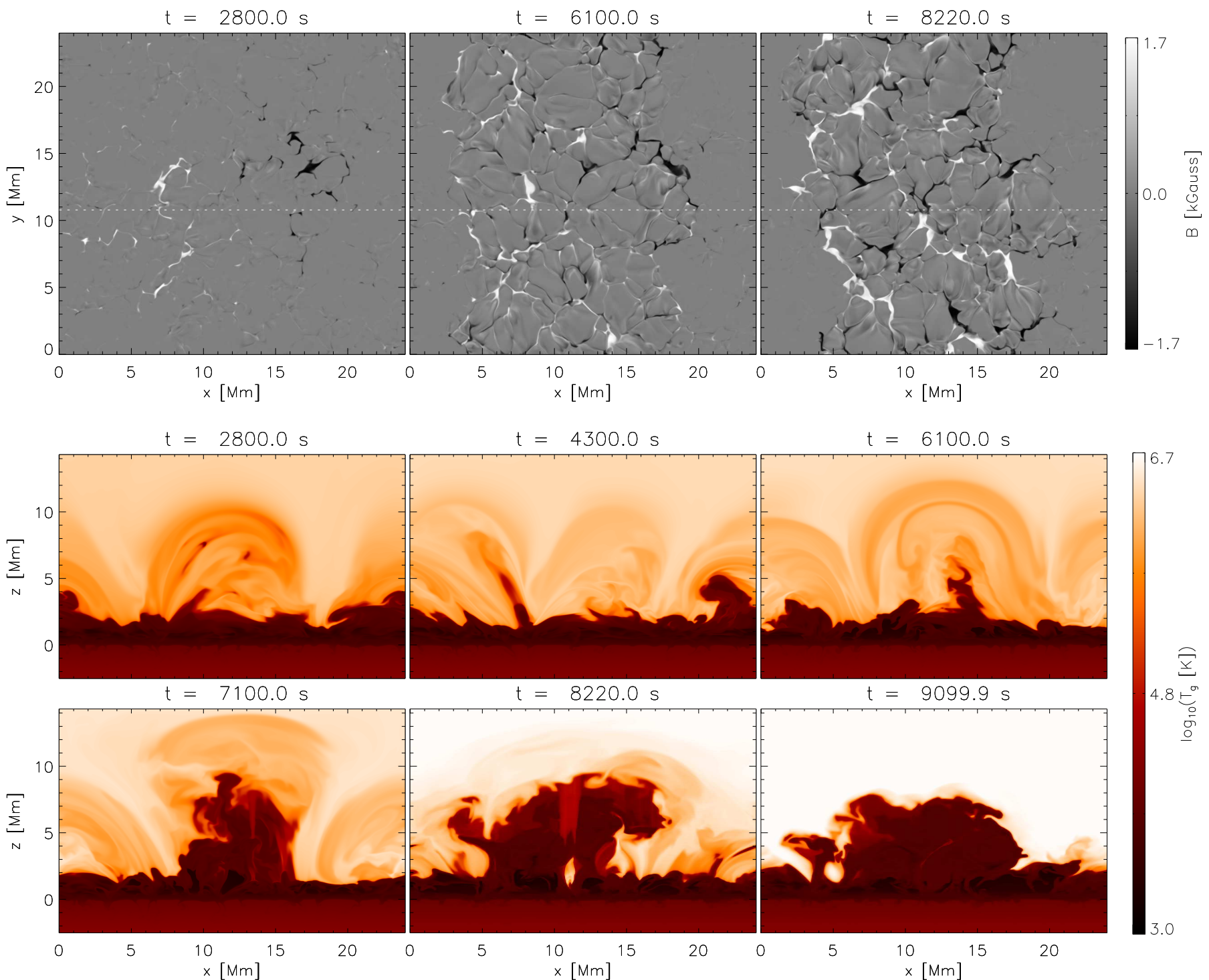

$t=90999 s$

Fig. 1. Vertical magnetic field $B_{z}$ in the photosphere at three separate times during the simulation (upper panels). The white dotted lines at $y=10.78 \mathrm{Mm}$ in the upper three panels show the location of the vertical cut in the $x z$-plane of the chromospheric and coronal temperature structure displayed in the panels below. These six panels show the evolution of the outer atmosphere as the magnetic bubble pierces the photosphere and expands through the chromosphere, pushing the corona upward, carrying cool material to large heights and heating the corona as the emergent and ambient field interact at large angle. The EB and UV burst described in the paper both occur in a period centered on $t=8200 \mathrm{~s}$. The UV burst visible in the lower middle panel lasts some $200 \mathrm{~s}$. The temperature rise of the EB is not easily visible in these panels because the temperature of EBs is far lower than that of the UV burst (but see Fig. 6). The EB occurs on the white dotted line around $x=11.5 \mathrm{Mm}$ near the center of the meeting opposite polarities that are visible in the upper right panel, and it lasts significantly longer than the UV burst. It starts at $t=7600$ and lasts at least $1200 \mathrm{~s}$ thereafter.

in the middle left panel of Fig. 1. Coronal field lines, for example, as outlined in emission in the Fe XII $19.51 \mathrm{~nm}$ line formed at 1.5 MK, show that the field lies at a $15^{\circ}$ angle to the $x$-axis.

The photosphere, as defined by the height where $\tau_{500 \mathrm{~nm}}=1$, is located at $z=0 \mathrm{~km}$. Above it, though corrugated, the initial chromosphere extends to $2 \mathrm{Mm}$, at which height we find the rapid temperature rise to coronal temperatures. Before the newly injected magnetic flux breaks through the photosphere, we find average coronal temperatures of $2 \mathrm{MK}$ at $10 \mathrm{Mm}$. Chromospheric and coronal temperatures and dynamics are maintained by acoustic waves and by footpoint braiding and subsequent nanoflares resulting from convective motions, as described in Carlsson et al. (2016) and Hansteen et al. (2015).

The model extends from $2.5 \mathrm{Mm}$ below the photosphere to 14.5 $\mathrm{Mm}$ above the photosphere in the vertical direction, and
$24 \mathrm{Mm}$ in both horizontal directions. This computational domain is covered by $768 \times 768 \times 768$ grid points, giving a horizontal grid size of $31.25 \mathrm{~km}$. The grid size varies in the vertical direction and is roughly $12 \mathrm{~km}$ from $-1.0 \mathrm{Mm}$ below the photosphere to $4.5 \mathrm{Mm}$ through the chromosphere. It slowly increases where the scale heights are larger, to $80 \mathrm{~km}$ at the upper boundary in the corona and to $20 \mathrm{~km}$ at the bottom convection zone boundary.

The calculations were carried out using the Bifrost code (Gudiksen et al. 2011). This code solves the magnetohydrodynamics (MHD) equations for a plasma in which the ionization state, for this specific model, is assumed to be in LTE. Energy source and sink terms include radiative losses; solving the equations of radiative transfer in four frequency bins and scattering for the optically thick photosphere and lower chromosphere; optically thin radiative losses based on recipes derived by 
Carlsson \& Leenaarts (2012) for the upper chromosphere, transition region, and corona; and thermal conduction along the magnetic field. The recipe for optically thin radiative losses includes back-heating by the $\operatorname{Ly} \alpha$ line and the Lyman-continuum, as described in Carlsson \& Leenaarts (2012).

\subsection{Spectral synthesis}

In order to compare our simulation with observed EBs and UV bursts, we synthesized spectral data in several often observed lines that are formed in the photosphere, chromosphere, transition region, and corona. These lines include $\mathrm{H} \alpha$, the $\mathrm{Ca}$ II $\mathrm{H} \& \mathrm{~K}$ and CaII $854.2 \mathrm{~nm}$ triplet line, lines of $\mathrm{Si}$ IV including the $139.376 \mathrm{~nm}$ line, and the coronal Fe XII $19.51 \mathrm{~nm}$ line.

The $\mathrm{H} \alpha$ scattering line requires a full 3D evaluation of the radiation field to obtain chromospheric features in the emergent intensities (Leenaarts et al. 2012). We therefore calculated the hydrogen lines using the MULTI3D code (Leenaarts \& Carlsson 2009). To model $\mathrm{H} \alpha$, we used a three-level plus continuum model atom of $\mathrm{H}$ I, where $\mathrm{Ly} \alpha$ is treated with a Doppler absorption profile in complete redistribution (Bjørgen et al. 2019).

Synthetic Ca II line profiles were calculated using the RH 1.5D code (Pereira \& Uitenbroek 2015). RH 1.5D solves the non-LTE radiative transfer problem by treating each column of a $3 \mathrm{D}$ model as a 1D atmosphere (1.5D approximation). Based on the RH code (Uitenbroek 2001), RH 1.5D allows partial redistribution effects, multiple species treated in non-LTE, and adding blending lines. To synthesize $\mathrm{Ca}$ II, we used a five-level plus continuum model atom in which the $\mathrm{Ca}$ II $\mathrm{H} \& \mathrm{~K}$ lines were treated in partial redistribution, while the infrared triplet lines were treated in complete redistribution.

Although the Si Iv $139.376 \mathrm{~nm}$ line, formed at transition region temperatures $(80 \mathrm{kK})$, is usually optically thin, the opacity of the line can sometimes become higher than unity. When the line was viewed from directly above, we therefore computed the $\mathrm{Si}$ Iv lines by solving the non-LTE equations of radiative transfer using the RH 1.5D code. We employed a 9-level atom, including 5 levels of Si III, the Si IV ground state, the upper levels of the 139.376 and $140.277 \mathrm{~nm}$ lines, and the Si v ground state. In addition, we included two more active atoms: a model atom with 15 levels of Si I and the ground state of Si II, and a 4-level model of Ni II-Ni III. This allowed us to derive the correct continuum intensity level for the Si Iv lines (the background opacity there is dominated by photoionization of $\mathrm{Si}$ I) and possible absorption in the Ni II $139.332 \mathrm{~nm}$ line, which is located at $93 \mathrm{~km} \mathrm{~s}^{-1}$ in the blue wing of Si Iv $139.376 \mathrm{~nm}$. The Ni II line is formed at $14 \mathrm{kK}$, which is much lower than the expected formation temperature of Si Iv. We did not include the Fe II lines that formed in the vicinity of $139.376 \mathrm{~nm}$ in this study. Moreover, the Si IV lines, both as viewed from above and from the side, were also calculated using an optically thin approximation with the same method as described below for Fe XII, and with data from version 8 of the CHIANTI atomic database (Del Zanna et al. 2015). Although differences are visible between the optically thin and optically thick results, the total intensities are qualitatively very similar.

The Fe XII $19.5 \mathrm{~nm}$ that forms around $1.5 \mathrm{MK}$ can be considered optically thin, and we calculated this line using collisional and ionization state data from version 8 of the CHIANTI database. The contribution to the intensity at frequency $v$ is given by

$\mathrm{d} I_{v}=\phi_{v}\left(u_{z}, T\right) A_{\mathrm{Fe}} n_{\mathrm{e}}^{2} g(T) \mathrm{e}^{-\tau} \mathrm{d} z$,

where $\phi_{v}$ is the (Gaussian) emission profile, $u_{z}$ is the vertical velocity, $T$ is the temperature, $A_{\mathrm{Fe}}$ is the abundance of iron, $n_{\mathrm{e}}$ is the electron density, $g(T)$ gives the excitation and ionization state of Fe xII, and $\mathrm{d} z$ is a distance element along the (vertical) line of sight. Because several regions of potential Fe XII emission are separated in places by several megameter of cool gas, we included absorption by neutral hydrogen and helium and singly-ionized helium in the $\mathrm{e}^{-\tau}$ absorption factor, following the recipes of Anzer \& Heinzel (2005), as done earlier and described in greater detail in De Pontieu et al. (2009). The total intensity in the line was found by integrating along the line of sight and in frequency across the line profile.

We assumed ionization equilibrium when we computed the Si IV and FexII lines. We included the effects of three-body recombination, which would allow Si Iv to be formed at temperatures down to $10-20 \mathrm{kK}$ at photospheric densities, but we did not see such temperatures near the dense plasma that forms the photosphere in this simulation (see, e.g., Rutten 2016).

\section{Results}

\subsection{Injection of the magnetic flux sheet}

A magnetic sheet, spanning $x=[4,18] \mathrm{Mm}$ and the full range along the $y$-axis, was injected at the bottom boundary $2.5 \mathrm{Mm}$ below the photosphere with a field strength of $B_{y}=2000$ Gauss and oriented in the $y$-direction. Through the action of buoyancy and convective upflows, the field rose through the upper convection zone and reached the photosphere after roughly one hour (at $t \approx 3500 \mathrm{~s}$ simulation time). On rising, the sheet was perturbed by convective motions; it was pulled down in locations of descending plumes of cold plasma and rose more slowly in upflow regions. It therefore reached the surface at slightly different times in discrete locations. On arriving at photospheric heights, the field was no longer buoyant and the upward motions stalled, but eventually, in locations where the field was strong enough, the field broke through the surface. The field in these locations rose rapidly and expanded into the chromosphere, carrying cool magnetized photospheric material upward. It was anchored to the photosphere in downdrafts where the field became nearly vertical (see Ortiz et al. 2014, for a detailed description of this phase). The state of the vertical field component in the photosphere is shown for two separate times in the middle and right panels of Fig. 1. Patches of opposite polarity are seen to pierce the photosphere in a fairly complex granular pattern that approximately fills the same horizontal extent as the horizontal size of the injected flux sheet. The field orientation is also close to what it was when it was injected $2.5 \mathrm{Mm}$ below. It forms an angle of approximately $75^{\circ}$ with the preexisting ambient field, which ensures large-angle reconnection as these fields, old and new, interact.

The field that rose into the chromosphere was strong, strong enough to push the preexisting ambient field upward and compress it. The newly emerging flux pulled cool photospheric and chromospheric material with it to great heights. This rise first halted some $10 \mathrm{Mm}$ above the photosphere, where further expansion was slowed by the preexisting field as the emerging and ambient fields attained about equal strengths of 30-50 Gauss at $10 \mathrm{Mm}$. This in contrast with the previous simulation of Archontis \& Hansteen (2014) and Hansteen et al. (2017), where the preexisting ambient field was very weak and was completely swept away by the newly emerging flux and fully removed the original corona. In the current simulation the preexisting ambient field was strong enough to hinder the expansion of the emerging bubbles and the ejection of the initial corona, which forces the bubbles to interact more strongly as both horizontal and 
vertical expansion is suppressed. This results in stronger and longer lasting current sheets that achieve lengths or heights of several thousand kilometers. The evolution of the emerging and expanding magnetic field is evident in the lower panels of Fig. 1, which show that the entire horizontal extent of the flux sheet rises and eventually impacts the corona, pushing it aside and filling the upper atmosphere with field and cool gas, but only up to a height of $10 \mathrm{Mm}$. The rising field expands from the photosphere in the form of cold magnetized bubbles, as described by Ortiz et al. (2014). As these bubbles expand, they eventually come into contact with each other, especially in regions where photospheric motions have brought their footpoints close together.

The interaction between the preexisting ambient field and the newly emerging field, which occurs near the top of the emerging bubble, essentially through high-angle reconnection, causes the coronal plasma temperatures at heights at and above $10 \mathrm{Mm}$ to increase significantly. These temperatures, at times of about $10 \mathrm{MK}$ as the flux rises and of about $4 \mathrm{MK}$ on longer timescales, are much higher than those generally achieved by low-angle reconnection that is driven solely by photospheric braiding (see, e.g., Galsgaard et al. 2007; Hansteen et al. 2015). However, we here concentrate on the high-angle reconnection, close to $180^{\circ}$, which occurs at lower heights, in the first few thousand kilometers above the photosphere, where oppositely directed magnetic fields that largely stem from the newly emerged field interact with each other: They heat and accelerate the cold plasma that is carried up by the emerging magnetic field.

\subsection{Formation of the current sheet and reconnection}

The oppositely directed magnetic field is brought together as the field expands into the upper chromosphere and by photospheric motions in many locations in the region that is covered by the emerging flux sheet. In particular, we concentrate on the fields in the vicinity of the center of the computational domain, located at $x=11 \mathrm{Mm}$ and $y=11 \mathrm{Mm}$, which is clearly visible in the center of the upper right panel of Fig. 1. At this location, two footpoints of opposite polarity, each the nexus of loop systems that are oriented roughly along the $y$-axis that span three to four granules, are brought into contact: A current sheet forms starting at $t=6000 \mathrm{~s}$. This current sheet strengthens with time, initially slanted but becoming nearly vertical in the next $1500 \mathrm{~s}$, stretching from the photosphere to almost $4 \mathrm{Mm}$ above. After formation, the current sheet maintains its shape and remains vertical and in the same location for at least the next $1000 \mathrm{~s}$, that is, to $t=9000 \mathrm{~s}$ (or slightly longer).

The topology of the magnetic field at the height of the UVburst emission is shown in Fig. 2, which also shows how oppositely directed fields are brought together and become the source of strong Joule heating and reconnection jets, which accelerate the plasma to high velocities. Above the reconnection region, we find emerged and previously reconnected field lines that have entered the outer solar atmosphere. They are oriented along the $y$-axis and are aligned with the injected field coming from below.

The current sheet leaves an imprint on the temperature of the rising cool gas and is visible as a heated region in the lower central panel of Fig. 1, which shows a $x z$-oriented vertical cut of the atmosphere at $y=10.78 \mathrm{Mm}$ and $t=8220 \mathrm{~s}$, as a "leaf-shaped" object. The form of the current sheet heating is also visible in the top panel of Fig. 2.

The general shape and variation in plasma parameters (temperature, magnetic field strength, vertical velocity, and $j^{2} / B^{2}$ ) in and around the current sheet are further shown in Fig. 3,

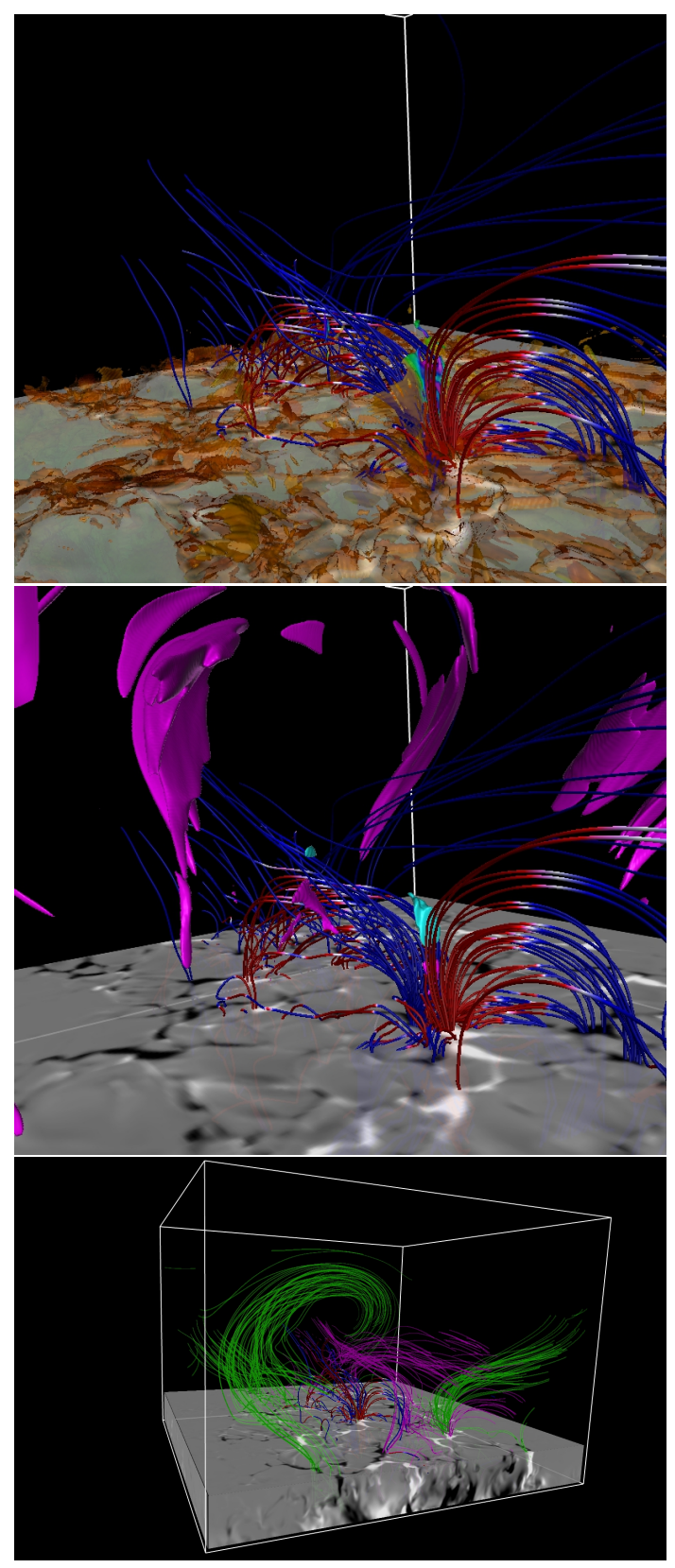

Fig. 2. Structure of chromosphere and corona above the flux cancellation site. Joule heating (top panel), up- and downflows in and around the current sheet (center panel), and magnetic field lines surrounding and above the current sheet. The green field lines in the lower panel outline the original ambient field that has been pushed upward and compressed by the emerging field (purple field lines) that is oriented largely in the $y$-direction (front to back in the figure). Below this field, we find a newly emerged field, drawn in red and blue to mark positive and negative $B_{z}$. The reconnection site driving the EB and UV burst is evident in the center of each panel. Reconnection heats and accelerates the plasma. Center panel: isosurfaces of $100 \mathrm{~km} \mathrm{~s}^{-1}$ (cyan) upward flows and $-60 \mathrm{~km} \mathrm{~s}^{-1}$ downward (purple) flows. Beige, brown, and green in the upper panel show the intensity of the Joule heating.

which shows a $y z$-oriented cut at $x=10.78 \mathrm{Mm}$ through the center of the sheet. A movie of its evolution, showing the same panels as in Fig. 3, is available online. The high-temperature phase of the current sheet, with temperatures well above $10 \mathrm{kK}$, which is responsible for the UV burst discussed below, only lasts for a limited $(200 \mathrm{~s})$ time. In contrast, the current sheet itself with associated reconnection events last much longer, and the 

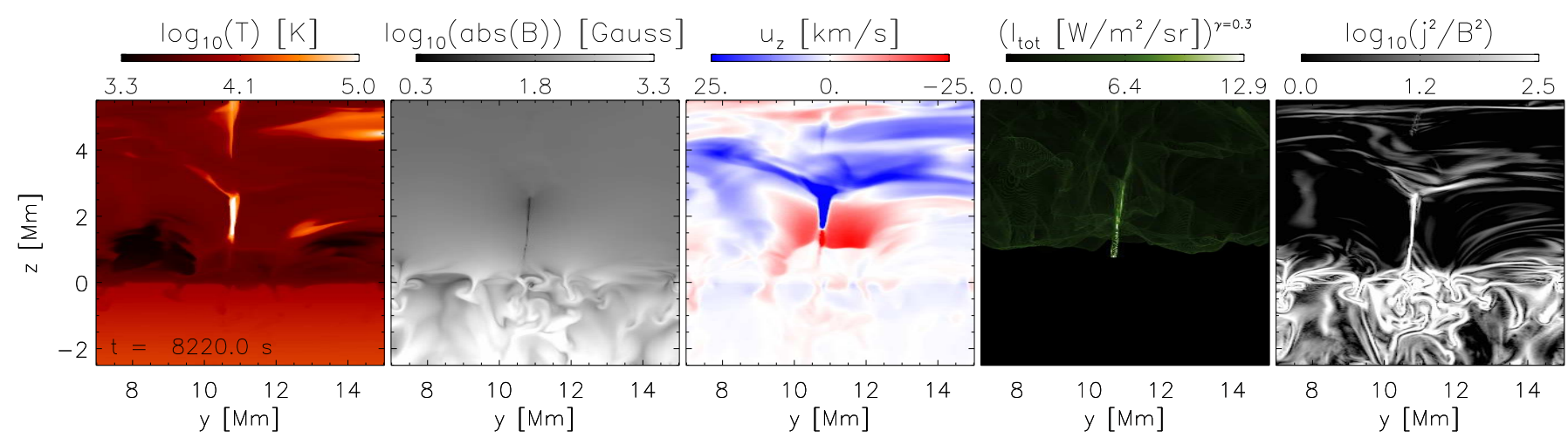

Fig. 3. Vertical cuts, at $x=10.78 \mathrm{Mm}$ in the $y z$ plane, of regions in the vicinity of the current sheet of Fig. 1 at $t=8220 \mathrm{~s}$ near the time of maximum intensity of the Si Iv lines. From left to right: log temperature, log total magnetic field strength, vertical velocity, Si Iv $139.376 \mathrm{~nm}$ emission, and current density $\left(j^{2} / B^{2}\right)$. The temperature is saturated at $\log (T)=5$ and the velocity at $u_{z}=[-25,25] \mathrm{km} \mathrm{s}^{-1}$, the maximum values of these quantities are higher than plotted here, as shown in Fig. 8. A movie showing the time evolution of the current sheet is available online.

temperature along the current sheet is at all times higher than ambient.

A number of bidirectional jets with velocities of up to $200 \mathrm{~km} \mathrm{~s}^{-1}$ are excited along the current sheet as reconnection commences. These jets heat the plasma, thus forming a thin vertical "leaf" of hot accelerated plasma that is located between the interacting bubbles of newly emerged cool gas. The activity engendered by the reconnection perturbs the plasma along the entire current sheet all the way from coronal heights $(3.5 \mathrm{Mm})$ and down to the photosphere. The movie of the current sheet evolution also shows that a number of plasmoids are generated and are accelerated either upward or downward during the lifetime of the current sheet.

\subsection{Ellerman bomb}

In the first few scale heights above the photosphere, this activity leads to the formation of an EB. The synthetic $\mathrm{H} \alpha$ spectra calculated using the MULTI3D code (Leenaarts \& Carlsson 2009) display many features that are the same as those that are observed. For example, in Fig. 4 the $\mathrm{H} \alpha$ emission is shown as seen from the side at an angle of $\mu=0.5$ : the wings of the profile form the typical EB moustaches, while the line core shows no brightening or evidence of heated gas. The EB forms a flamelike structure that is clearly visible in spectroheliograms made in the red and blue wings of $\mathrm{H} \alpha$ (the red wing is shown in Fig. 4). This structure extends to $1.2 \mathrm{Mm}$ above the photosphere.

The line core, shown in the right panel of Fig. 4, shows extensive fibrils, some have about the length of the computational box, $20 \mathrm{Mm}$, but no evidence of brightening directly above the site of the EB. The two loop systems that meet and are the source of the EB are visible in the center of the right panel of Fig. 4. They stretch toward greater and lower $y$-values from their meeting point close to $y=11 \mathrm{Mm}$ ?

When viewed from directly above, the EB region is bright in $\mathrm{H} \alpha$ in the line core as well, but this is due to emission that is formed much higher in the atmosphere, at least $5 \mathrm{Mm}$ above the photosphere: The cool canopy above the current sheet is heated by Ly $\alpha$ and Ly-continuum radiation from the hot plasma of the current sheet below, raising the temperature of the canopy plasma significantly, as is visible in the lower middle panel of Fig. 1 and in the left panel of Fig. 3. This relatively hightemperature gas excites some $\mathrm{H} \alpha$ emission in the region that is heated above the current sheet. This heating at large heights directly above the reconnection region is probably a result of the
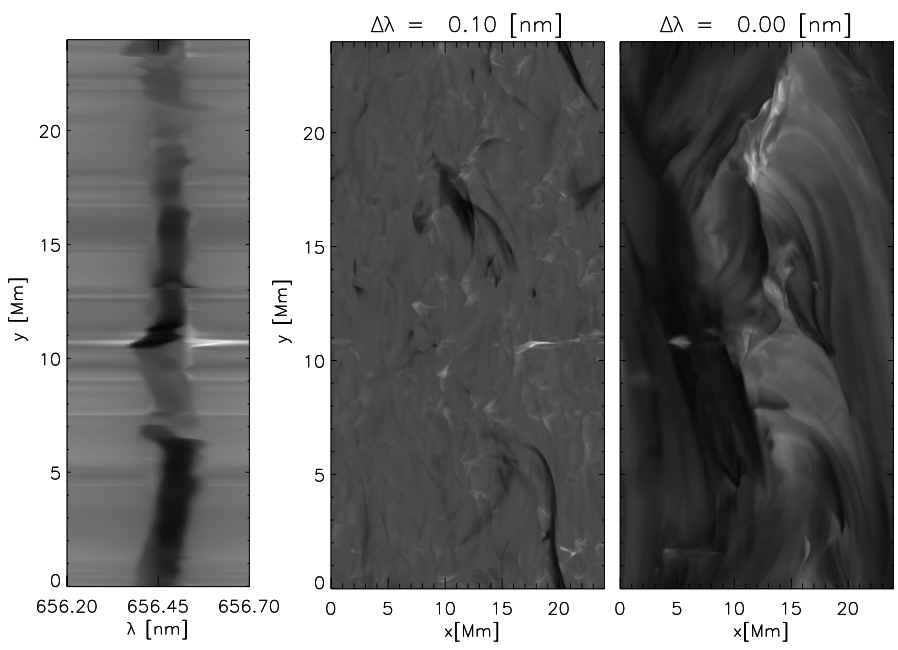

Fig. 4. $\mathrm{H} \alpha$ spectroheliograms at $+0.1 \mathrm{~nm}$ and at line center (center and right panels, respectively) at a viewing angle of $\mu=0.5$. The EB is located at $x=17 \mathrm{Mm}$. Because of the projection effect of looking from the side, this corresponds to $x=11$ when viewed from directly above. The line profile over the EB, shown in the left panel, is located at $[x, y]=$ $[17,11] \mathrm{Mm}$ and is evident in the line wings.

method we chose for the coronal back-radiation and raises the temperature more than what would occur on the real Sun. The heating does not affect the evolution or diagnostics of the EB or UV burst.

Swedish 1-meter Solar Telescope observations (Ortiz et al., in prep.) show that the wing of the Ca II $854.2 \mathrm{~nm}$ line can be a very good proxy for the existence of EBs, with enhanced emission that is colocated and roughly cotemporal with bright $\mathrm{H} \alpha$ wing enhancement. These observations in the blue wings of both the $\mathrm{Ca}$ II $854.2 \mathrm{~nm}$ and $\mathrm{H} \alpha$ lines also show dark, presumably cool, surges that occasionally emanate from the site of EB emission. We find that the EB in this location shows brightening, starting at time $t=7500 \mathrm{~s}$ and lasting for at least $1200 \mathrm{~s}$ thereafter. This enhanced emission, $0.0735 \mathrm{~nm}$ in the blue wing of the Ca II $854.2 \mathrm{~nm}$ triplet line, is shown in Fig. 5, which also shows emission in the line core and in the shape of the line profile. Similar as for $\mathrm{H} \alpha$, the cores of the $\mathrm{Ca}$ II H \& Kand the Ca II $854.2 \mathrm{~nm}$ triplet lines show the loops that emanate from the vicinity of the current sheet as narrow thin fibrils that stretch toward higher and lower $y$-values, approximately in the $y$-direction. The EB is visible as a narrow band of emission in the line wing, and it is also 

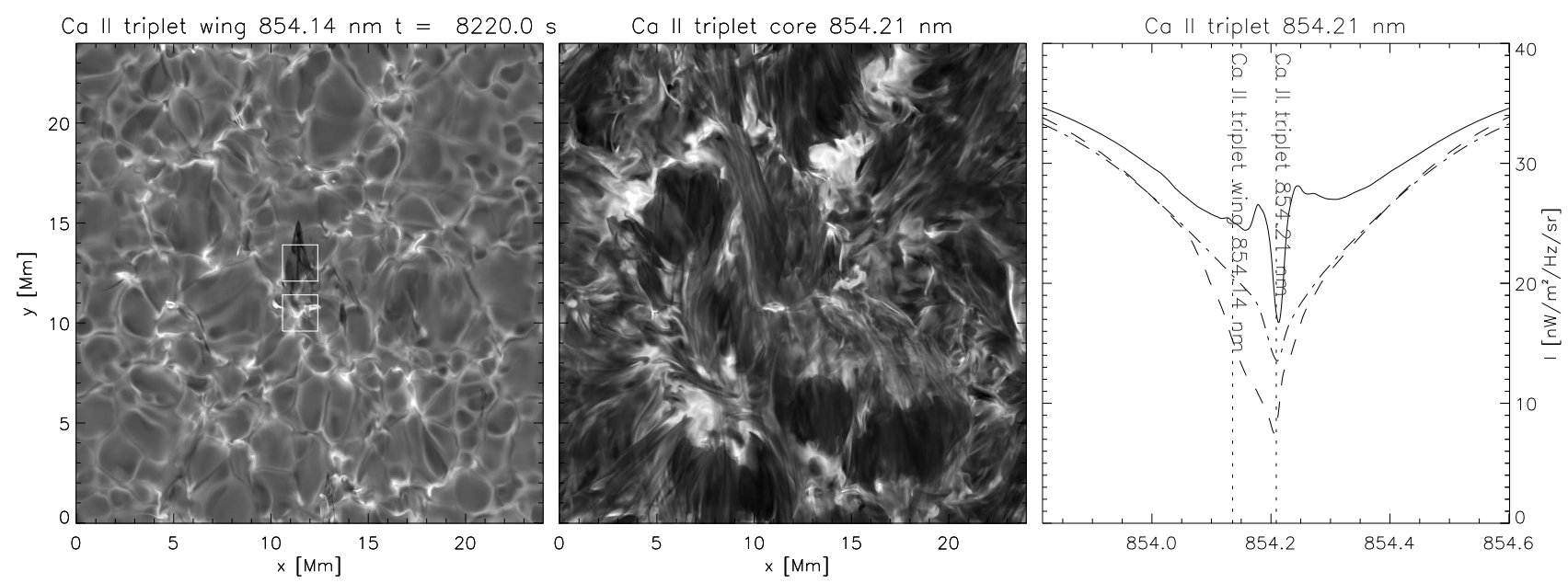

Fig. 5. Ca II $854.2 \mathrm{~nm}$ line filtergrams showing the blue line wing and line core, as well as the line profile, at the time of a small cool surge. Right panel: line profiles for the EB region (solid line), the cool surge (dashed line), and the average spectrum (dash-dotted line). The EB and cool surge regions we used to construct the profiles are indicated by the boxes in the left panel.

visible at the time of this image $(t=8220 \mathrm{~s})$ in the line core, although this emission is partially covered by overlying longer fibrils. Apparently rising from the EB location, about parallel with the positive $y$-axis, a small cool surge is seen to be accelerated along the field lines, or loops, that form the cool portion of the canopy. Signs of the surge are visible for some minutes (from $t=8140 \mathrm{~s}$ to $t=8300 \mathrm{~s}$ ). The line profile of the EB region shows enhanced emission in both wings and self-reversal in the line core. The profile of the small surge shows absorption that extends to $0.15 \mathrm{~nm}\left(35 \mathrm{~km} \mathrm{~s}^{-1}\right)$ in the blue wing of the Ca II $854.2 \mathrm{~nm}$ line.

The structure of the atmosphere in the vicinity of the current sheet that produced the EB is shown in Fig. 6. In the location where positive and negative polarities meet, that is, $x=[11,12] \mathrm{Mm}, y \approx 10.5 \mathrm{Mm}$, we see heightened temperatures. The current sheet is the site of high-velocity downflows and hotter-than-average chromospheric temperatures that extend all the way down to the photosphere. The temperature around the current sheet is about $7500 \mathrm{~K}$, at least $1500 \mathrm{~K}$ warmer than the ambient average chromosphere. (On the other hand, temperatures below the photosphere in the location of the current sheet are lower than ambient.) We find large downflows of $25 \mathrm{~km} \mathrm{~s}^{-1}$ or more, which are rapidly decelerated in the region down to $500 \mathrm{~km}$. Even closer to the photosphere and below, downflows of about $10 \mathrm{~km} \mathrm{~s}^{-1}$ fill the area in and around the current sheet. These higher temperatures and high downflow velocities are concentrated in a narrow region with horizontal extent of some $700 \mathrm{~km}$ where field lines of opposite polarity meet, that is, in the region of the current sheet. In the fluxemerging region outside of the current sheet, the chromosphere included in the magnetic bubbles does not generally have highly supersonic velocities, nor a temperature rise to coronal temperatures until the canopy is reached at some $9 \mathrm{Mm}$ above the photosphere.

An exception to this is found in Fig. 6, which shows that we sometimes also find areas of high downflow velocities, $10 \mathrm{~km} \mathrm{~s}^{-1}$ or more, in the chromosphere outside the current sheet, such as the circular region near $[x, y]=[11,9.5] \mathrm{Mm}$, but without a corresponding temperature rise. Temperatures of $<3000 \mathrm{~K}$ are seen in regions that contain large unidirectional fields. These may be the sites of convective collapse, with no associated temperature rise and thus no EB.
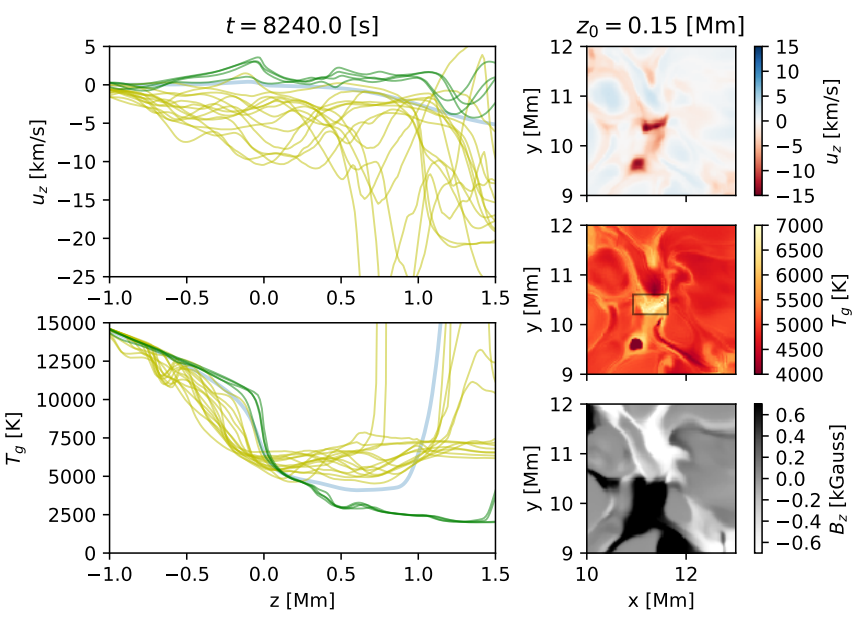

Fig. 6. Velocity, temperature, and field strength in the $x y$-plane $z=$ $150 \mathrm{~km}$ above the photosphere (rightmost three panels) in the vicinity of the current sheet that generated the EB described in the text. The current sheet is located in the center of the small box shown in the center of the right central panel. Two leftmost panels: yellow lines: vertical velocity and temperature as functions of height in vertical columns in this small box, averaged over tiles of $150 \mathrm{~km}(5 \times 5$ grid zones $)$. Green lines: average of the same variables some distance away from the current sheet, near $[x, y]=[10,9] \mathrm{Mm}$. Blue lines: average velocity and temperature as a function of height in the entire computational domain.

\subsection{The UV burst}

While the EB is well established already at $t=7500 \mathrm{~s}$, the UV burst is not visible before $t=8040 \mathrm{~s}$ (although there is an earlier UV burst in about the same location that is part of the same flux system) at which point the temperature in the current sheet at heights from $700 \mathrm{~km}$ up to $3500 \mathrm{~km}$ rises rapidly, increasing from $10000 \mathrm{~K}$ to more than $1 \mathrm{MK}$ within $20 \mathrm{~s}$ or less. This event occurs in the same horizontal location and as part of the same magnetic flux system that produces the EB described in the previous section. As described below, this reconnection event leads to a increase in the Si Iv emission of more than two orders of magnitude above average total intensities. Above the current sheet, the plasma remains cool, $<10000 \mathrm{~K}$ all the way up to $9 \mathrm{Mm}$ above the photosphere, where it rapidly rises to coronal values. 

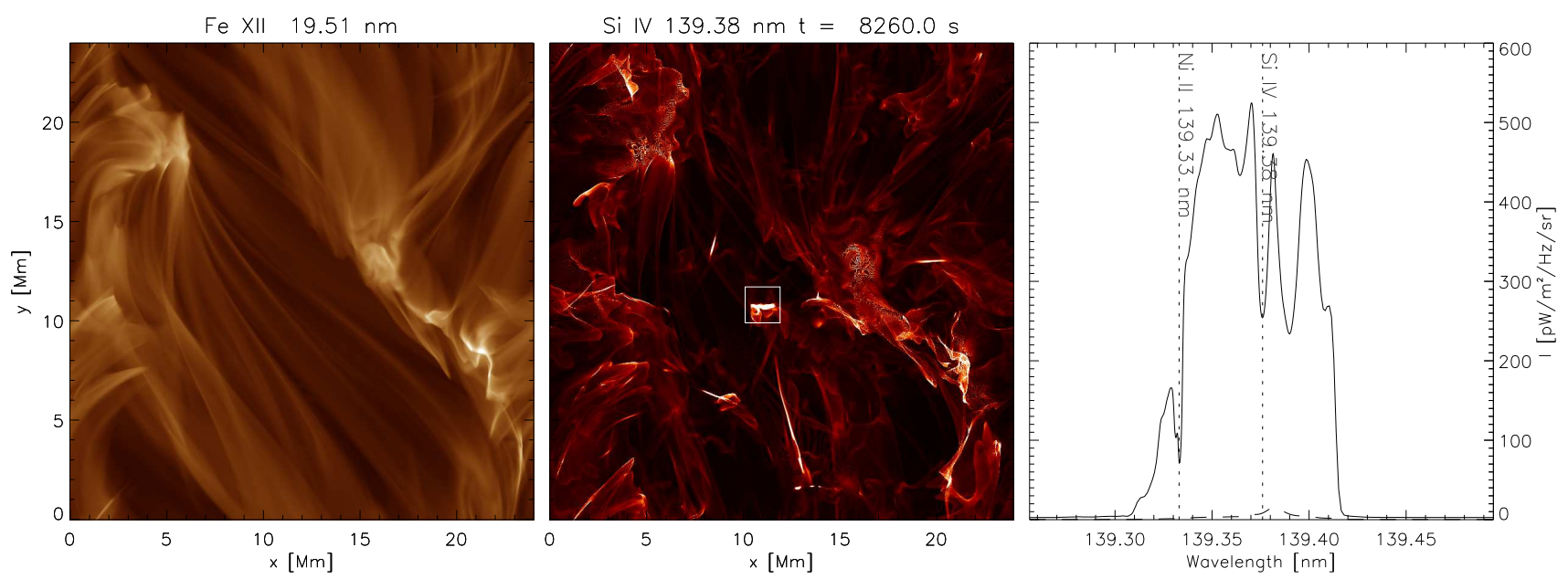

Fig. 7. Fe XII $19.5 \mathrm{~nm}$ and $\mathrm{Si}$ Iv $139.376 \mathrm{~nm}$ line total line intensities as well as the line profile of the Si iv line (where the full line shows the profile of the UV burst and the dashed line represents the average spectrum). The location of the UV burst is indicated by the box in the central panel.

The plasma is at times heated to above $1 \mathrm{MK}$, and in principle, emission might also be expected in the Fe XII $19.51 \mathrm{~nm}$ line from the current sheet. However, this turns out not to be the case when the line is calculated as optically thin using data from the CHIANTI package (Landi et al. 2006). Figure 7 shows that emission in the Fe xII line appears to consist of long strands outlining the canopy above the flux-emerging region, showing mainly the long $(20 \mathrm{Mm})$ strands or loops that lie above the shorter interacting loop systems described above in Sect. 3.3. No additional Fe XII emission is visible in the vicinity of the current sheet. This is not because the current sheet does not emit in the Fe XII $19.51 \mathrm{~nm}$ line, but arises because absorption from the neutral hydrogen and helium gas overlies the current sheet, which has high opacity that is included in the calculation and is found to absorb the emission from this line. Running the calculation without absorption does indeed show bright emission emanating from the site of the current sheet. Recently, Guglielmino et al. (2019) have found Fe xII emission in the (usually very weak) $134.9 \mathrm{~nm}$ line observed with IRIS, cospatial with Si IV UV burst emission. This provides observational evidence for coronal temperatures in this event.

As shown in the central panel of Fig. 7, the Si iv $139.376 \mathrm{~nm}$ line has copious emission in the vicinity of the current sheet, up to two or more orders of magnitude brighter than the average intensity of this line in the computational domain. We find that the average line profile has a total intensity of $2 \mathrm{~W} \mathrm{~m}^{-2} \mathrm{sr}^{-1}$, an average redshift of $10 \mathrm{~km} \mathrm{~s}^{-1}$, and a width of $25 \mathrm{~km} \mathrm{~s}^{-1}$ (which is reasonably close to thermal). The situation is found to be radically different in the area around the current sheet: As seen from above, the total intensity of this line reaches very high values in a thin region with a width of some hundred kilometers, sometimes extending to several hundred kilometers, stretching along the extent of the current sheet "leaf", roughly 500-700 km. As noted and shown in Fig. 1, an extended region of cool gas lies above the current sheet. Because continuum absorption is weak, this cool gas is relatively transparent near $140 \mathrm{~nm}$, but we find line absorption in the Si Iv $139.376 \mathrm{~nm}$ line when it becomes broad enough to be blended with cool lines such as the Ni II $139.332 \mathrm{~nm}$ line, which lies $93 \mathrm{~km} \mathrm{~s}^{-1}$ blueward of the Si Iv $139.376 \mathrm{~nm}$ line center. We show in Fig. 7 the line profile as calculated with RH and including the opacity of Ni II. The Si IV line has attained high intensity and an extremely non-Gaussian shape with a width of more than $200 \mathrm{~km} \mathrm{~s}^{-1}$. Absorption in the $\mathrm{Ni}$ II line is visible (the
Si IV $140.277 \mathrm{~nm}$ line profile has an almost identical shape, but shows no intensity dip at $93 \mathrm{~km} \mathrm{~s}^{-1}$ blueward of the line center). This shows that enough cool material lies above the current sheet to produce the UV burst to both hinder emission in the Fe XII $19.51 \mathrm{~nm}$ line and show absorption for a cool line such as Ni II $139.332 \mathrm{~nm}$. The latter line is at times shifted $5-10 \mathrm{~km} \mathrm{~s}^{-1}$ blueward, which is an indication that the bubble of cool gas carried by the emerging magnetic flux is still partially ascending at this time.

We consider the temperature and velocity structure of the upper portion of the current sheet in more detail. We find very high velocities throughout the current sheet at heights from $700 \mathrm{~km}$ to $3.5 \mathrm{Mm}$. The upper part of the current sheet has upflow velocities of up to $200 \mathrm{~km} \mathrm{~s}^{-1}$ in single-pixel columns, while the lower portion of the current sheet shows downflows of more than $-50 \mathrm{~km} \mathrm{~s}^{-1}$. This is clear from Fig. 8, which shows $5 \times 5$ gridpoint averages of the vertical velocities and temperatures in and in the vicinity of the current sheet. Reconnection in the current sheet drives bidirectional flows, and the highest temperatures are in locations where these flows are decelerated in shocks, near $z=1 \mathrm{Mm}$ for the downflows and $z=3 \mathrm{Mm}$ for the upflows. This plasma, heated to well above $100000 \mathrm{~K}$ and moving in both directions at high velocity, gives rise to the extreme emission in transition region lines, such as shown above for the Si IV lines around $140 \mathrm{~nm}$. Figure 3, showing the total intensity of the Si Iv $139.376 \mathrm{~nm}$ line as seen from the side (and as calculated as optically thin using data from the CHIANTI atomic data package), reaches high values, up to two orders of magnitude above ambient, along the entire upper part of the current sheet from some $700 \mathrm{~km}$ to $3 \mathrm{Mm}$ above the photosphere.

\section{Discussion and conclusions}

The simulations presented in this paper show that the conditions required to generate many of the diagnostics associated with both EBs and UV bursts can occur naturally as a result of the emergence of a fairly strong but untwisted magnetic flux sheet into an atmosphere that contains a preexisting magnetic field of similar strength. The successfully synthesized diagnostics include $\mathrm{H} \alpha$ wing enhancements in the form of "moustaches" and flame-like structures in $\mathrm{H} \alpha$ spectroheliograms. We also found brightenings in the wings of the Ca II triplet lines (as well as 

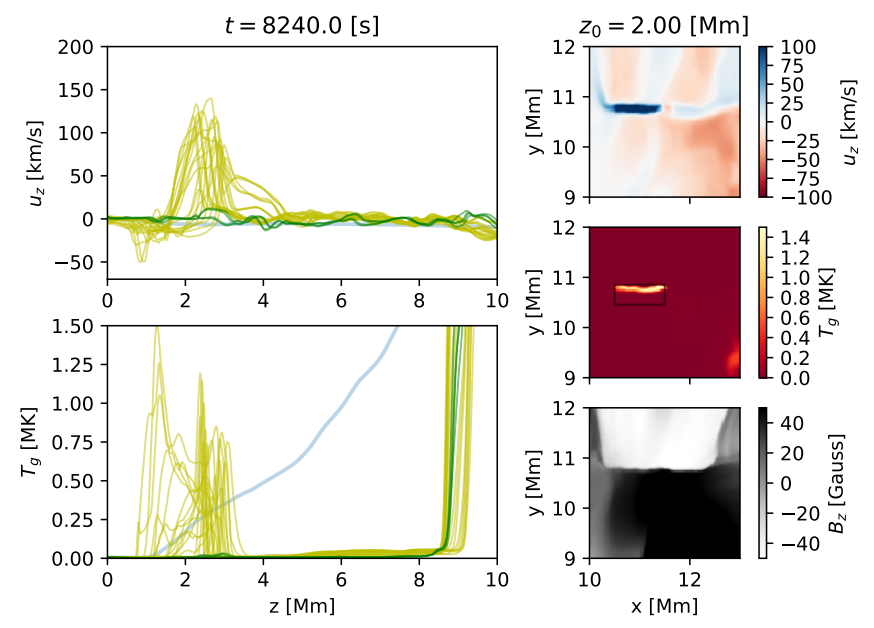

Fig. 8. Velocity, temperature, and field strength in the $x y$-plane $z=$ $2 \mathrm{Mm}$ above the photosphere (rightmost three panels) in the vicinity of the current sheet that generated the UV burst described in the text. The current sheet is located in the center of the small box shown in the center of the right central panel. Two leftmost panels: yellow lines: vertical velocity and temperature as functions of height in vertical columns in this small box, averaged over tiles of $150 \mathrm{~km}(5 \times 5$ grid zones $)$. Green lines: average of the same variables some distance away from the current sheet, near $[x, y]=[10,9] \mathrm{Mm}$. Blue lines: average velocity and temperature as a function of height in the entire computational domain.

occasionally the line core), and the acceleration of cool surges was seen in the $\mathrm{Ca}$ II triplet wings.

We did not include phenomena that may be important to the physics or diagnostics of the chromosphere, such as ambipolar diffusion and nonequilibrium ionization. In addition, the magnetic diffusivity of the numerical model is, by necessity, several orders of magnitude higher than what is the case on the real Sun. However, the very good fit between the synthetic diagnostics and the observations and the nonexistent change in these diagnostics when the resolution was increased by nearly a factor 2 compared to the Hansteen et al. (2017) study, make us confident that this model is qualitatively correct.

Colocated and cotemporal with the EB discussed in this paper, we also found that the Si Iv lines near $140 \mathrm{~nm}$ were greatly enhanced, by two to three orders of magnitude. They also showed extremely non-Gaussian line profiles and broadening, which is indicative of velocities of up to $>200 \mathrm{~km} \mathrm{~s}^{-1}$. The Fe XII $19.51 \mathrm{~nm}$ emission, on the other hand, shows no reaction to the presence of hot dense gas in the first few megameter above the photosphere. The general picture that we obtain here is that when EBs and UV bursts occur simultaneously as part of the same structure, EBs are formed in the first few hundred to one thousand kilometers of the upper photosphere and lower chromosphere, while the UV burst is formed up to several chromospheric scale heights higher in the chromosphere, over an extended region. In the specific example considered in this paper, the EB is formed from the photosphere up to $1200 \mathrm{~km}$ above, while the UV burst is formed at heights between $700 \mathrm{~km}$ and $3 \mathrm{Mm}$ above the photosphere. The vertically elongated and flame-like shape of the EB is very similar to what is observed (i.e., Watanabe et al. 2011), while the observations of UV bursts are not as clear: Young et al. (2018) write that “... a burst may appear with extended structure (jet, fibril, loop) connected to it, but these are typically less bright. The burst itself may also appear spatially extended into one direction "flame", but remains $<2$ arcsec". It remains to be determined whether this is compatible with the emission that this simulation produced. The observations indicate that between $10 \%$ and $20 \%$ of EBs show signatures of UV bursts (Grubecka et al. 2016; Tian et al. 2016; Ortiz et al., in prep.). We found some examples in the simulation described here, but not enough to carry out a statistical analysis. Furthermore, such an analysis should probably take into consideration a more realistic topology of the emerging field, for example, from an emerging active region- This is beyond the scope of this study.

The source of this emission is the current sheet that is formed by the collision of two oppositely directed bundles of magnetic flux that are pushed together by photospheric motions. The current sheet stretches from the photosphere some $3 \mathrm{Mm}$ into the chromosphere while spanning 1-2 Mm horizontally. It is the site of vigorous reconnection, accelerating bidirectional jets of plasma to velocities of about the Alfvén speed; tens of $\mathrm{km} \mathrm{s}^{-1}$ near the photosphere up to several hundred $\mathrm{km} \mathrm{s}^{-1}$ at heights of $1 \mathrm{Mm}$ or more. While the $\mathrm{H} \alpha$ and Ca II wing emission is formed mainly in the first few hundred kilometers above the photosphere, we also find absorption by fast-moving gas at much greater heights, as seen in the blue wing of Ca II in Fig. 5, for example, where the cool surge is comprised of gas $2 \mathrm{Mm}$ above the photosphere. This is presumably gas accelerated by the Lorentz force, as first described by Yokoyama \& Shibata (1996), or with additional acceleration by pressure gradient forces as the upward-moving gas is pressed against a "wall" of strong field, as discussed by Nóbrega-Siverio et al. (2016). The main result of reconnection is the heating of plasma in the vicinity of the current sheet, either directly through Joule heating or resulting from the thermalization of the kinetic energy of the gas that is accelerated by reconnection jets as they are slowed down in shocks. In the first few hundred kilometers above the photosphere, the gas is heated $2-3000 \mathrm{~K}$ above ambient temperatures, but at greater heights, where radiative losses are less efficient, the plasma can at times attain temperatures of $1 \mathrm{MK}$ or more for several hundred seconds.

The current sheet is located in a large bubble of emerging magnetic field, carrying with it cool gas from the photosphere. This cool gas has high opacity in typically photospheric or cool lines, but also in the continua of hydrogen and helium shortward of $91.1 \mathrm{~nm}$, which is relevant for the EUV Imaging Spectrometer for Hinode and the Advanced Imaging Assembly on the Solar Dynamics Observatory observables such as He II $30.4 \mathrm{~nm}$, Fe XII $19.51 \mathrm{~nm}$, and Fe IX $17.1 \mathrm{~nm}$. Thus we find emission from the current sheet suppressed in the Fe XII $19.51 \mathrm{~nm}$ line, and we find line absorption by Ni II $139.332 \mathrm{~nm}$ line in the wing of Si Iv 139.376 when this line becomes broad enough.

During the course of the simulation, several regions of opposite magnetic field polarity were brought together as a result of photospheric motions. These led to reconnection in or just above the photosphere, in the middle to upper chromosphere, or in some cases in both when the topology of the interacting magnetic bubbles allowed the formation of a current sheet with a length of several megameters. We therefore expect either EBs, UV bursts or both to be generated readily as a result of flux emergence. When both phenomena occur simultaneously or nearly so, as described in this paper, it may be possible to observe a certain shift in location of the EB and UV burst depending on the orientation of the current sheet and the viewing angle; such shifts would presumably be easier to confirm when they are observed close to the solar limb.

We therefore see no compelling reason to posit that UV bursts occur in the photosphere. Instead we propose that the 
observations point to a scenario where the chromosphere has become vastly bloated with slowly rising $\left(10 \mathrm{~km} \mathrm{~s}^{-1}\right)$ cool fairly dense gas, up to $10 \mathrm{Mm}$ or more. Further, that this cool gas supplies the necessary opacity to explain absorption in lines such as $\mathrm{Ni}$ II and Mn I, and in the continua of hydrogen and neutral and singly-ionized helium. These provide narrow absorption bands in the Si IV and Mg II line wings, and continuum absorption suppressing the evidence for intense heating in the AIA bands such as $30.3,17.1$, and $19.3 \mathrm{~nm}$ in the lower regions of the expanded active region chromosphere.

Acknowledgements. This research was supported by the Research Council of Norway through grant 170935/V30, through its Centres of Excellence scheme, project number 262622. Computing time has come through grants from the Norwegian Programme for Supercomputing, as well as from the Pleiades cluster through the computing project s1061, from the High End Computing (HEC) division of NASA. The 3D radiative transfer computations were performed on resources provided by the Swedish National Infrastructure for Computing (SNIC) at the High Performance Computing Center North (HPC2N) at Umea University and the PDC Centre for High Performance Computing (PDC-HPC) at the Royal Institute of Technology in Stockholm. Some images were produced by VAPOR (www . vapor . ucar . edu), a product of the Computational Information Systems Laboratory at the National Center for Atmospheric Research.

\section{References}

Anzer, U., \& Heinzel, P. 2005, ApJ, 622, 714

Archontis, V., \& Hansteen, V. 2014, ApJ, 788, L2

Bjørgen, J. P., Leenaarts, J., Rempel, M., et al. 2019, A\&A, submitted

Carlsson, M., \& Leenaarts, J. 2012, A\&A, 539, A39

Carlsson, M., Hansteen, V. H., Gudiksen, B. V., Leenaarts, J., \& De Pontieu, B. 2016, A\&A, 585, A4

Del Zanna, G., Dere, K. P., Young, P. R., Landi, E., \& Mason, H. E. 2015, A\&A, 582, A56

De Pontieu, B., Hansteen, V. H., McIntosh, S. W., \& Patsourakos, S. 2009, ApJ, 702, 1016

De Pontieu, B., Title, A. M., Lemen, J. R., et al. 2014, Sol. Phys., 289, 2733
Ellerman, F. 1917, ApJ, 46, 298

Fang, C., Hao, Q., Ding, M.-D., \& Li, Z. 2017, Res. Astron. Astrophys., 17, 031 Galsgaard, K., Archontis, V., Moreno-Insertis, F., \& Hood, A. W. 2007, ApJ, 666,516

Grubecka, M., Schmieder, B., Berlicki, A., et al. 2016, A\&A, 593, A32

Gudiksen, B. V., Carlsson, M., Hansteen, V. H., et al. 2011, A\&A, 531, A154

Guglielmino, S. L., Young, P. R., \& Zuccarello, F. 2019, ApJ, 871, 82

Gupta, G. R., \& Tripathi, D. 2015, ApJ, 809, 82

Hansteen, V., Guerreiro, N., De Pontieu, B., \& Carlsson, M. 2015, ApJ, 811, 106

Hansteen, V. H., Archontis, V., Pereira, T. M. D., et al. 2017, ApJ, 839, 22

Judge, P. G. 2015, ApJ, 808, 116

Kim, Y.-H., Yurchyshyn, V., Bong, S.-C., et al. 2015, ApJ, 810, 38

Landi, E., Del Zanna, G., Young, P. R., et al. 2006, ApJS, 162, 261

Leenaarts, J., \& Carlsson, M. 2009, in The Second Hinode Science Meeting: Beyond Discovery-Toward Understanding, eds. B. Lites, M. Cheung,

T. Magara, J. Mariska, \& K. Reeves, ASP Conf. Ser., 415, 87

Leenaarts, J., Carlsson, M., \& Rouppe van der Voort, L. 2012, ApJ, 749, 136

Ni, L., Lin, J., Roussev, I. I., \& Schmieder, B. 2016, ApJ, 832, 195

Ni, L., Lukin, V. S., Murphy, N. A., \& Lin, J. 2018, ApJ, 852, 95

Nóbrega-Siverio, D., Moreno-Insertis, F., \& Martínez-Sykora, J. 2016, ApJ, 822, 18

Ortiz, A., Bellot Rubio, L. R., Hansteen, V. H., de la Cruz Rodríguez, J., \& Rouppe van der Voort, L. 2014, ApJ, 781, 126

Pariat, E., Aulanier, G., Schmieder, B., et al. 2004, ApJ, 614, 1099

Pereira, T. M. D., \& Uitenbroek, H. 2015, A\&A, 574, A3

Peter, H., Tian, H., Curdt, W., et al. 2014, Science, 346, C315

Priest, E. R., Chitta, L. P., \& Syntelis, P. 2018, ApJ, 862, L24

Rutten, R. J. 2016, A\&A, 590, A124

Rutten, R. J., Vissers, G. J. M., Rouppe van der Voort, L. H. M., Sütterlin, P., \& Vitas, N. 2013, J. Phys. Conf. Ser., 440, 012007

Syntelis, P., Priest, E. R., \& Chitta, L. P. 2019, ApJ, 872, 32

Tian, H., Xu, Z., He, J., \& Madsen, C. 2016, ApJ, 824, 96

Toriumi, S., Katsukawa, Y., \& Cheung, M. C. M. 2015, ApJ, 811, 137

Uitenbroek, H. 2001, ApJ, 557, 389

Vissers, G. J. M., Rouppe van der Voort, L. H. M., Rutten, R. J., Carlsson, M., \& De Pontieu, B. 2015, ApJ, 812, 11

Warren, H. P., Winebarger, A. R., \& Brooks, D. H. 2012, ApJ, 759, 141

Watanabe, H., Vissers, G., Kitai, R., Rouppe van der Voort, L., \& Rutten, R. J. 2011, ApJ, 736, 71

Yokoyama, T., \& Shibata, K. 1996, PASJ, 48, 353

Young, P. R., Tian, H., Peter, H., et al. 2018, Space Sci. Rev., 214, 120 